Revue des patrimoines

4 | 2004

Les réseaux de la villégiature

\title{
Les réseaux d'investissement dans le thermalisme au XIXe siècle en France
}

Jérôme Penez

\section{(2) OpenEdition}

Journals

Édition électronique

URL : http://journals.openedition.org/insitu/1665

DOI : 10.4000/insitu.1665

ISSN : 1630-7305

Éditeur

Ministère de la Culture

Référence électronique

Jérôme Penez, "Les réseaux d'investissement dans le thermalisme au XIXe siècle en France », In Situ [En ligne], 4 | 2004, mis en ligne le 01 mars 2004, consulté le 07 septembre 2020. URL : http:// journals.openedition.org/insitu/1665; DOI : https://doi.org/10.4000/insitu.1665

Ce document a été généré automatiquement le 7 septembre 2020

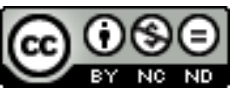

In Situ Revues des patrimoines est mis à disposition selon les termes de la licence Creative Commons Attribution - Pas d'Utilisation Commerciale - Pas de Modification 4.0 International. 


\section{Les réseaux d'investissement dans le thermalisme au XIXe siècle en France}

Jérôme Penez

Figure 1

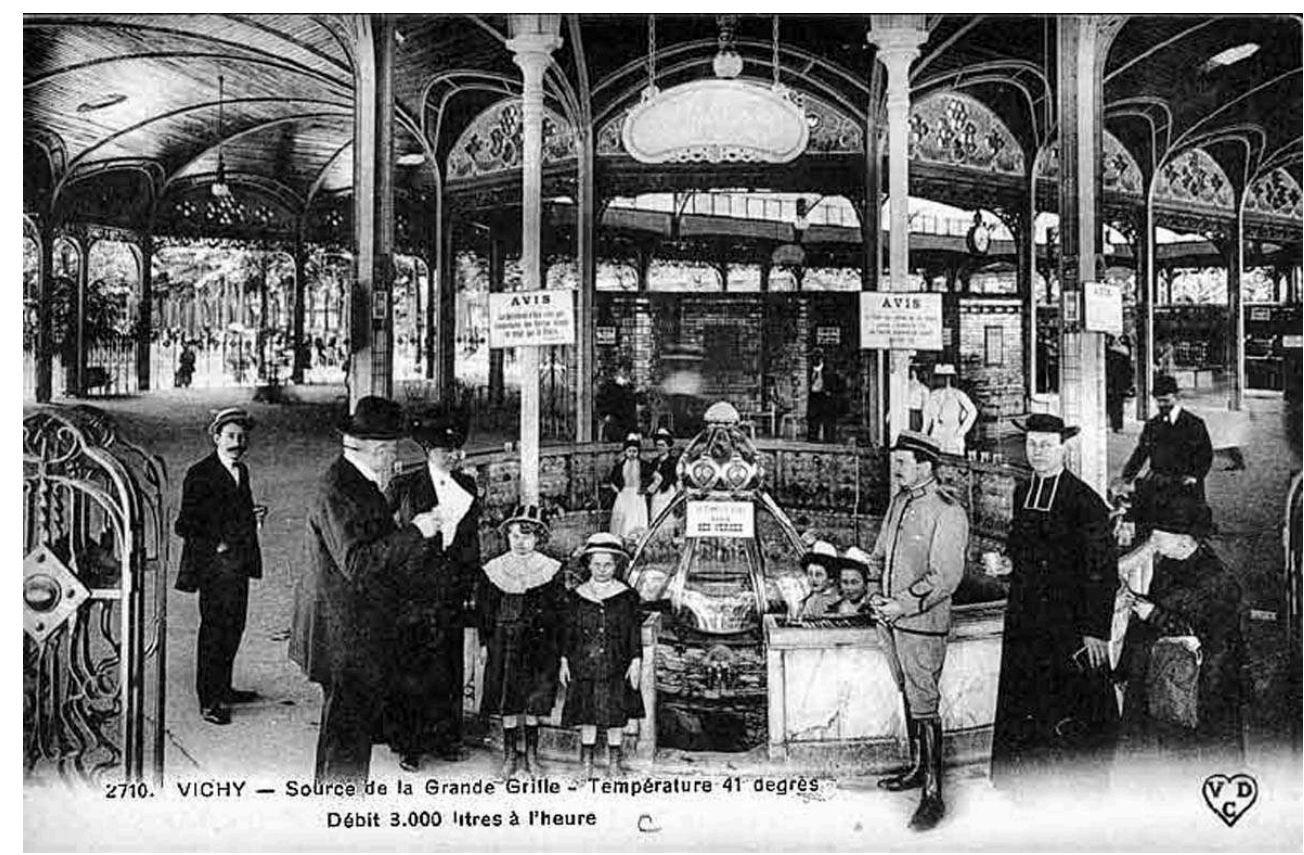

Vichy (Allier). Le hall des Sources puis palais des Sources abritant les buvettes des sources de la

Grande-Grille, Chomel, Lucas et Mesdames. Carte postale, début XXe s., coll. B. Toulier. Extrait de Villes d'eaux, Paris : Imprimerie Nationale Editions/Dexia, 2002, p. 73.

$1 \quad{ }^{1}$ UJ'ai prévu les constructions suivantes :

2 Un petit bâtiment de type industriel, où se feraient l'arrivée principale de l'eau, la mise en bouteilles, les diverses opérations commerciales et administratives. 

à sept cent milles francs. (...) Je crois que la source a une valeur. Mais il n'est pas raisonnable d'en attendre des miracles. Il dépend de nous que de toute façon l'affaire ne soit pas mauvaise. Comment cela? En faisant de la source une raison d'être honorable, le point d'attraction autour duquel nous développerons un pays de villégiature. (...) Le site et les environs sont plaisants. Une petite ville d'agrément à l'usage de la région parisienne peut y pousser aussi bien qu'ailleurs. Il suffit qu'elle trouve un peu plus de motifs de prendre racine là qu'ailleurs. Ce léger supplément de motifs (...) c'est notre source qui est appelée à le fournir.

7 Il est normal que vous vous demandiez que l'intérêt propre je poursuis. Aucun qui soit indépendant des vôtres.

Comme je crois à l'avenir de cette affaire, je veux ne rien devoir qu'à son succès. Je ne vous demande donc ni de me racheter mes options, ni de me payer des commissions, ni même de me rembourser mes frais. Tout ce que j'ai en mains, je le verse à la constitution de la Société.

9 L'apport n'est pas négligeable, je le sais. J'espère que vous m'offrirez un siège au Conseil, parce que je crois que je l'ai mérité, et que je puis y rendre des services. Voilà, messieurs. A vous de décider $»^{2}$.

10 Ainsi s'exprime Haverkamp, l'un des héros des Hommes de bonne volonté de Jules Romains, dans un discours prononcé devant des actionnaires potentiels de la société qu'il envisage de fonder pour gérer la future station thermale de la Celle-les-Eaux, en région parisienne. Haverkamp n'est pas jusqu'alors un spécialiste de la "gestion thermale ", il n'a même jamais dirigé d'entreprises ; la source dont il est question il ne l'a pas découverte, elle existait déjà. La source d'eau minérale n'est pas pour lui un moyen de soulager les souffrances d'autrui, ni le moyen de développer l'activité des populations qui habitent à proximité, ce qu'il recherche c'est la rentabilité et la reconnaissance de ses qualités dans le milieu des affaires, de ses capacités de gestionnaire. Tout "naturellement», Haverkamp décide de créer une société " capitaliste ». Au début du XXe siècle, à l'époque où se déroule l'action du roman de Jules Romains, le recours au capitalisme et à des actionnaires est la meilleure solution pour créer une entreprise, celle qui apparaît être la plus « logique ».

11 Bien sûr, si Haverkamp avait disposé de suffisamment d'argent peut-être n'aurait-il pas eu besoin de faire appel à d'autres capitaux que les siens, mais la présence d'autres financiers permet de minimiser les investissements, de ne pas risquer son argent seulement, et de pouvoir compter sur des appuis différents et multiples, en partant du principe que les actionnaires ont intérêt à ce que la station thermale réussisse.

Haverkamp a bien compris que l'élément indispensable et essentiel, c'est la source d'eau minérale, il a aussi très bien saisi que le griffon n'est qu'un des éléments d'une ville d'eaux, les distractions, les hôtels, les villas sont également indispensables, et doivent être financés. 
13 L'exemple d'Haverkamp est celui d'un entrepreneur privé qui se lance dans le thermalisme, mais ce dernier n'est pas uniquement géré par le "privé »: l'Etat, les départements, les communes prennent une part active à l'administration des sources hydrominérales et de l'environnement thermal. C'est une des particularités du thermalisme que de posséder des acteurs économiques aux statuts différents qui cohabitent pour la gestion des sources thermales.

L'exemple de la fondation « romanesque » de la station thermale de La Celle-les-Eaux est révélateur d'une évolution dans la gestion du thermalisme : le passage de plus en plus fréquent du public au privé; de l'Etat, des collectivités locales aux entreprises privées, au capitalisme, aux sociétés par actions.

Jules Romains n'est pas le seul écrivain à s'être intéressé à l'évolution du thermalisme et plus précisément à l'économie du thermalisme. Quelques décennies plus tôt, Guy de Maupassant dans son roman « Mont Oriol » décrit l'histoire de la création d'une station thermale.

16 En parallèle à une histoire d'amour romanesque, c'est toute la machinerie thermale qui est décrite, du financement à la concrétisation d'une ville d'eau. S'inspirant de l'histoire "réelle» de la ville de Châtel-Guyon, Maupassant montre les rouages économiques du thermalisme.

17 L'intérêt des écrivains français pour l'évolution du thermalisme s'explique par le constat selon lequel depuis le milieu du XIXe siècle, l'exploitation des sources minérales connaît un véritable engouement, c'est une véritable « fièvre thermale » qui s'empare de la France, et plus largement de l'Europe.

18 Les villes d'eaux fleurissent sur le territoire français, surtout localisées à proximité et au cœur des massifs montagneux. Au début du XXe siècle, elles sont plus d'une centaine de stations réputées, attirant plusieurs centaines de milliers de baigneurs, de buveurs d'eau, de touristes. Il existe également environ une centaine d'autres stations qui ne connaissent que de très loin les fastes des villes d'eaux et qui se contentent de « survivre » souvent avec une clientèle locale et très réduite.

19 Comme l'a constaté Jules Romains, créer une station thermale ce n'est pas seulement exploiter l'eau minérale, c'est également mettre en place un environnement propice composé à la fois de loisirs, de distractions, sans omettre bien entendu l'aspect médical, raison d'être du thermalisme. Cette bivalence entre loisirs et maladie se retrouve dans les infrastructures thermales: établissements thermaux, parcs, casinothéâtre, hôtels.

20 Construire de pareilles villes, qui cherchent à être des villes idéales, nécessite de très lourds investissements, surtout à la fin du XIXe siècle, alors que - notamment après la guerre de 1870 - la concurrence est exacerbée entre les villes d'eaux à l'échelle nationale, mais aussi européenne.

21 Un dilemme se pose alors aux gestionnaires, aux investisseurs du thermalisme : être rentable, créer une affaire prospère sans spéculer sur l'état des malades ni créer des lieux où les débordements des plaisirs soient indécents. Rentabilité, médecine, loisirs, maladie sont autant d'éléments qu'il est difficile de faire cohabiter durant le XIXe siècle. Il est intéressant de s'interroger sur l'envers du décor thermal, des fastes de la vie thermale ou des échecs thermaux, pour évoquer les investissements effectués dans les villes d'eaux. Avant tout, il convient d'avoir à l'esprit la diversité du thermalisme : diversité du fait de la période évoquée, l'ensemble du XIXe siècle, mais surtout diversité 
en raison des différents types de gestion, des différentes tailles des villes d'eaux, de leur localisation différente, de leur réussite variable. Cette variété explique qu'il n'existe pas réellement de schéma préconçu menant au succès thermal, ni de véritables réseaux d'investissements au sens actuel du terme.

Quel que soit le statut du propriétaire ou du gestionnaire, ce sont bien souvent des hommes qui ont été à l'origine du développement économique du thermalisme, seuls ou en groupe, ce sont des individus qui sont pour une grande part les « héros » heureux ou malheureux des aventures thermales. Même dans le cas de propriété ou de gestion publique des sources, le facteur "humain » intervient, peut-être moins que dans les entreprises privées, mais il ne doit pas être négligé comme le prouvent les actions successives du préfet Raymond de Carbonnières et du médecin inspecteur Bertrand dans la gestion de la station thermale départementale du Mont-Dore.

A l'inverse, de nombreux échecs peuvent être imputables aux hommes qui n'ont pas su ou qui n'ont pas voulu valoriser le potentiel thermal. Il serait injuste de considérer que les «naufrages thermaux » soient seulement à mettre sur le compte du facteur humain, d'autres éléments peuvent entrer en jeu ; il paraît inconcevable que la gestion thermale ne se solde que par des réussites, la France ne peut pas compter des dizaines de «Vichy » ou d'« Aix-les-Bains », le potentiel de la fréquentation ne le permet pas.

Les stations thermales qui attirent le plus de visiteurs sont généralement des villes d'eaux "publiques", pourtant leur nombre est faible par rapport à l'ensemble des exploitations thermales: les "stations d'Etat» sont seulement sept, un nombre constant pour le XIXe siècle, ce qui ne signifie pas qu'aucune modification n'ait eu lieu. En 1831, l'Etat met aux enchères les sources de Pougues-les-Eaux, tandis qu'en 1853, il acquiert celles de Luxeuil ; celles appartenant aux départements et aux communes sont une cinquantaine, un chiffre qui varie peu au cours du XIXe siècle, tandis que celles appartenant à des particuliers ou à des sociétés capitalistes sont une centaine au début du XIXe siècle et le double en 1900. Il semble que la «fièvre thermale " soit surtout le fait des investisseurs privés.

Des rapprochements entre les types de propriété existent: Vichy, station dont les principales sources sont comprises dans le domaine public, est gérée à partir de 1853 par une puissante société capitaliste.

Les changements de propriétaires et de gestionnaires sont nombreux au XIXe siècle, preuve à la fois du dynamisme du secteur thermal et de la difficulté de pouvoir réellement rentabiliser et développer une activité fortement liée aux aléas de la demande. Quel que soit le propriétaire des sources hydrominérales, public ou privé, la gestion des sources ne doit pas être déficitaire. Même l'Etat se doit de faire des bénéfices avec ses griffons. Dans les stations thermales gérées par l'Etat, lorsque les pertes s'alourdissent les décisions de cession se précisent, voire se concrétisent.

Le thermalisme n'apparaît donc pas comme un « élément clé » de l'économie nationale suscitant un intérêt prioritaire pour le gouvernement et pouvant engendrer indéfiniment des déficits.

\section{L'Etat : entre régie et affermage}

28 L'Etat, législateur et aussi gestionnaire, est un acteur du thermalisme à double visage. C'est le deuxième aspect qui nous intéresse davantage ici. Il gère en effet plusieurs 
sources thermales en France directement sous forme de régie ou indirectement par l'intermédiaire de fermiers : Vichy, Aix-les-Bains (station gérée d'abord par l'Etat sarde avant 1860, puis par l'Etat français), Plombières, Néris, Bourbon-l'Archambault, Bourbonne-les-Bains, Luxeuil à partir de 1853, Pougues-les-Eaux jusqu'en 1831, Provins jusqu'en 1840.

29 L'Etat s'intéresse depuis longtemps à l'exploitation directe des stations thermales. En 1808, Napoléon $1^{\mathrm{er}}$, sur proposition du préfet des Pyrénées Chazal, décide de créer une administration centrale des eaux thermales. Les stations de Bagnères-de-Bigorre, de Cauterets, de Luz, de Barèges, de Capvern, de Labassère, des Eaux-Bonnes, des EauxChaudes, de Cambo, de Luchon ont été regroupées et gérées par l'administration. L'application de cette décision ne fut que très partielle et peu à peu les sources pyrénéennes ont eu des modes de gestion différents, pour la plupart communaux.

30 Au XIXe siècle, l'Etat n'a ni les moyens financiers, ni la volonté de prendre en main la gestion de toutes les sources thermales, il se concentre sur certaines d'entre elles. L'Etat français possède les stations thermales les plus importantes. Cette possession n'est pas entière, bien sûr, ce ne sont que les sources qui sont sa propriété, mais à partir de ce précieux liquide, le gouvernement français, qu'il soit monarchique, impérial ou républicain a su créer les villes d'eaux les plus renommées du territoire français, parmi lesquelles se trouve le fleuron du thermalisme français : Vichy.

31 L'Etat est devenu propriétaire de sources, plus par hasard que par une volonté délibérée de prendre en main le potentiel thermal de la France. La Révolution et l'Empire ont été à l'origine de la plus forte croissance du domaine hydrominéral étatique : Plombières, Bourbonne-les-Bains, Néris, Bourbon-l'Archambault et Vichy par confiscation pour ces trois dernières stations des possessions des Bourbons qui étaient sous l'Ancien régime déjà comprises dans le domaine royal. 
Figure 2

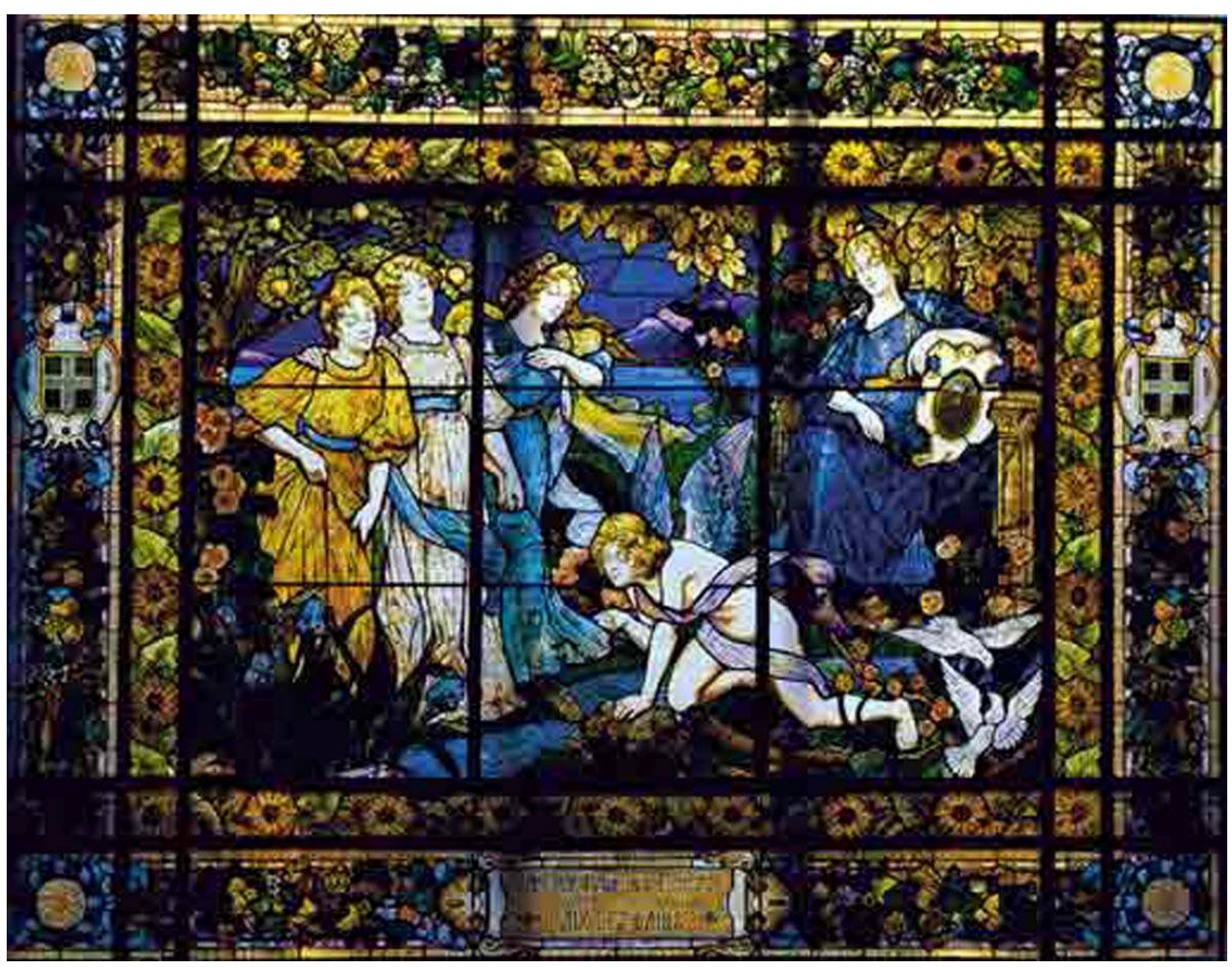

Aix-les-Bains (Savoie). Détail d'un vitrail de la galerie des Glaces du Grand Cercle : « L'Amour [qui] puise ses forces aux sources bienfaisantes d'Aix-les-Bains. Par Jacques Galland, 1897. Photo. Caroline Rose. Extrait de Villes d'eaux, Paris : Impr merie Nationale Editions/Dexia, 2002, p. 103.

Toutes les sources possédées par l'Etat ne connaissent pas un destin aussi glorieux que Vichy ou Aix-les-Bains (fig. $\mathbf{n}^{\circ}$ 2) ; par exemple les sources de Médagues dans le Puyde-Dôme, affermées pendant une grande partie du XIXe siècle, ne sont fréquentées que par quelques dizaines de malades.

L'Etat gère aussi des hôpitaux civils ou militaires dans les stations thermales. Les hôpitaux militaires participent directement à l'organisation thermale, surtout dans des stations comme Barèges ou Bourbonne-les-Bains.

Pour gérer son domaine thermal, l'Etat a souvent hésité entre affermage et mise en régie, entre gestion directe et appel au capitalisme. La différence entre affermage et régie est due généralement à la volonté ou non de l'Etat d'essayer de développer l'exploitation. Souvent, faute de volonté d'investir directement, l'Etat fait appel à des investisseurs privés pour créer les infrastructures thermales nécessaires au lancement d'une station. Toutefois, ce cas de figure n'existe que dans le second XIXe siècle comme le prouve l'exemple de Plombières. Dans la première moitié du XIXe siècle à Plombières, c'est l'affermage qui est choisi, en faveur de modestes propriétaires habitant Plombières. Aucun investissement particulier ne leur est demandé, si ce n'est le simple entretien des bâtiments. L'affermage des salons de détente est également attribué à des Plombinois.

En 1857, l'affermage est attribué à une compagnie plus puissante : la compagnie pour l'exploitation des sources et des établissements thermaux de Plombières, compagnie créée par la volonté de Napoléon III et qui doit impérativement comporter des actionnaires originaires de la région vosgienne. 

membre du conseil général des Vosges, maire de la ville de Remiremont, Joseph Parisot, maire de Plombières, et également des industriels comme Charles Demande, maitre de forges en Haute-Saône et membre du conseil général de Haute Saône, et Victor de Pruines également maitre de forges et conseiller général des Vosges. A cette liste s'ajoutent quelques membres de professions libérales, médecins, notaires, pharmaciens... Les actions, d'une valeur de 500 francs, sont prévues pour produire $5 \%$ d'intérêt par an, taux garanti par l'Etat. Napoléon III fait un don de 55000 Francs à la nouvelle société. La concession du domaine thermal est de 80 ans, une durée inhabituellement longue, ce qui montre la volonté de l'Etat d'aider la nouvelle entreprise. En échange la nouvelle société doit construire un ensemble complet d'infrastructures thermales : des thermes, un casino, des hôtels, un parc..., le tout dans un délai de 3 ans. L'Etat ne reçoit aucune ferme, excepté les impôts, tels que contributions foncières pendant dix ans, au-delà, il percevra un pourcentage des bénéfices. Afin d'aider la nouvelle société, l'Etat prend à sa charge les travaux de captage des eaux minérales et l'assainissement de la ville à hauteur de 345000 francs et se porte garant des emprunts successifs réalisés par la société.

Ce puissant parrainage et ces aides ne parviennent pas à rendre la société rentable, en grande partie en raison des investissements exorbitants en infrastructure, les actionnaires ne recevront jamais plus de $2 \%$ d'intérêts et souvent le chiffre est plus proche de zéro.

Figure 3

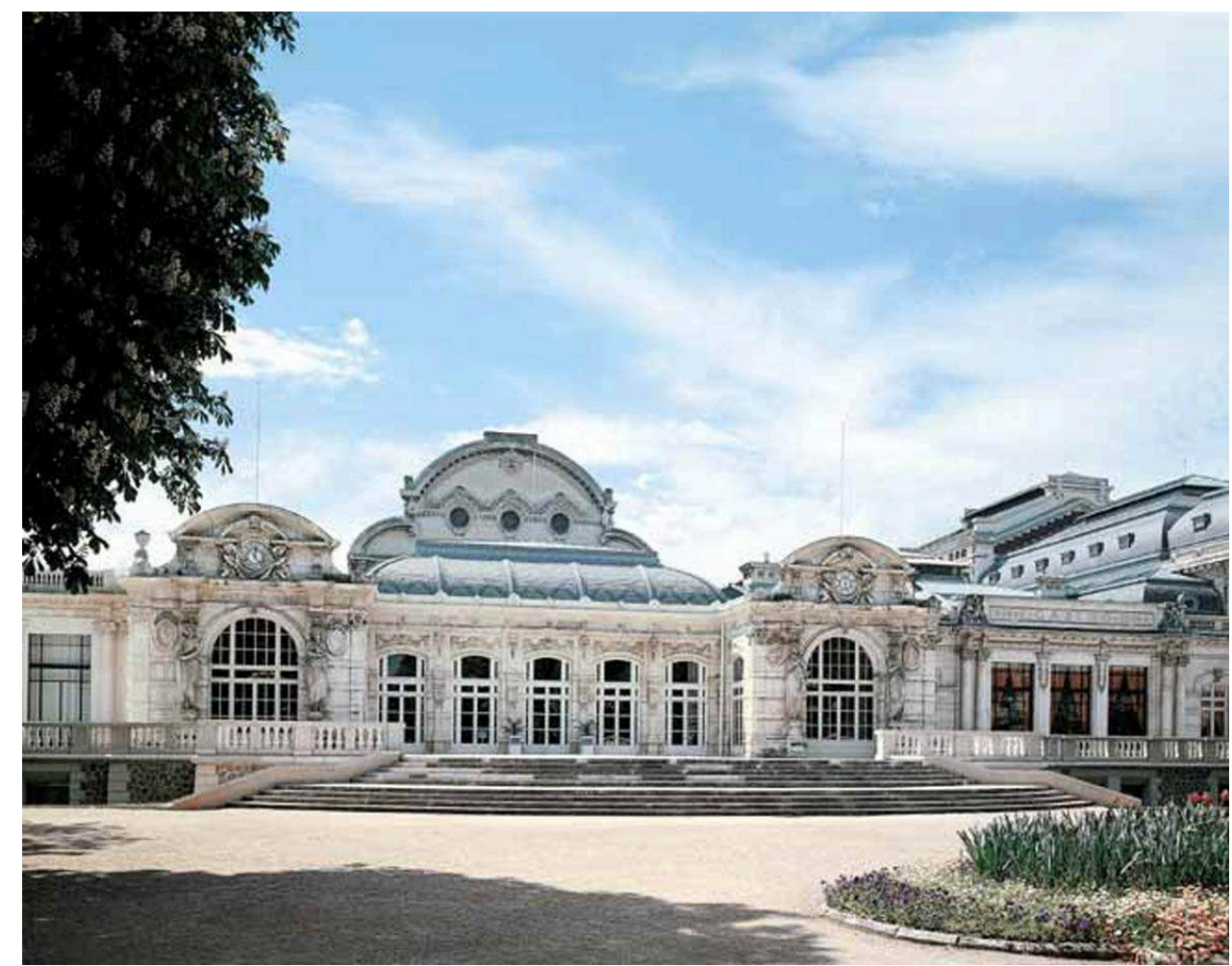

Vichy (Allier). Casino-Théâtre, façade principale du premier casino édifié entre 1863 et 1865 par l'architecte Charles Badger à la suite de la visite de Napoléon III. Photo. Caroline Rose. Extrait de Villes d'eaux, Paris : Imprimerie Nationale Editions/Dexia, 2002, p 105. 
situation est différente à Vichy, la « reine des villes d'eaux ». L'Etat, après avoir hésité pendant la première moitié du XIXe siècle entre affermage et régie, décide en 1853 d'opter pour l'affermage. Comme à Plombières, la décision est prise en raison d'importants investissements indispensables en infrastructure sous peine de voir la clientèle bouder la station. L'affermage a lieu en faveur d'une société créée principalement par Auguste Lebobe, entrepreneur de travaux publics parisien, député de l'arrondissement de Meaux et administrateur des chemins de fer du Nord et Georges Callou, entrepreneur de travaux publics (fig. $\mathbf{n}^{\circ} \mathbf{3}$ ).

39 A ces deux principaux actionnaires, qui détiennent 390 des 400 actions de la société, se sont joints le docteur Arnal, médecin de Napoléon III et Jean-Frédéric Possoz, maire de Passy. Le capital social est important, 2 millions de francs. Les charges imposées aux fermiers sont lourdes: verser 100000 francs par an de ferme, verser à l'hôpital de Vichy 5 centimes par litre d'eau expédié, effectuer des travaux pour 1 million de francs, acquérir trois sources concurrentes, accepter la présence et le contrôle d'un commissaire du gouvernement. En dépit de charges élevées, qui s'expliquent par la situation déjà bien établie de la station vichyssoise, la société prospère.

Plusieurs modifications et augmentations de capital sont effectuées sous le Second Empire, notamment lors du décès d'Auguste Lebobe et du départ d'Arthur Callou. En 1863, une liste des actionnaires de la Compagnie fermière des eaux thermales montre une domination forte des Parisiens: sur 76 actionnaires 63 habitent Paris, "seulement" trois résident à Vichy (un pharmacien possesseur de 50 actions, un banquier détenteur de 20 actions et un "propriétaire » avec 20 actions). De nombreux actionnaires sont notés comme étant des " propriétaires » ou des « négociants » : 35 sur les 76 actionnaires. Les médecins sont peu nombreux: 5. Les autres participants à l'aventure vichyssoise sont essentiellement des membres des professions libérales, architectes, avocats. La Compagnie fermière possède une assise sociale assez large, recrutant dans un "vivier» parisien d'hommes d'affaires à la recherche de fructification d'un capital. Cette situation est donc différente de celle de Plombières où les acteurs locaux ont pris la direction et composent l'essentiel de la société fermière.

A Aix-les-Bains, l'Etat français décide pendant une grande partie du XIXe siècle d'adopter la mise en régie, à part un léger intermède lors de la tentative de Cavour d'affermer le domaine thermal appartenant à l'époque au royaume de PiémontSardaigne. 


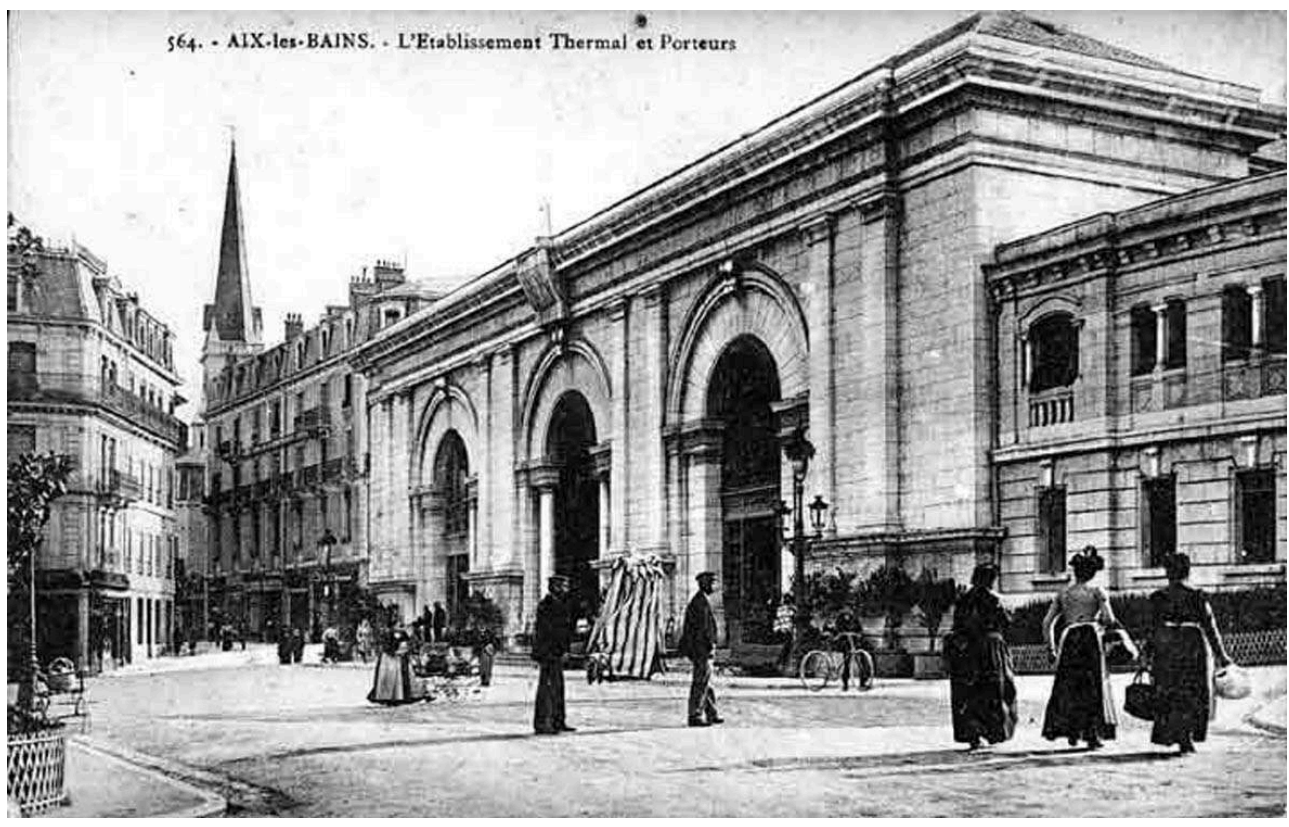

Aix-les-Bains (Savoie). Etablissement thermal, façade antérieure. Conçu en 1854 et construit de 1857 à 1861 par l'architecte Charles Pellegrini. Carte postale, début XXe s., coll. B. Toulier. Extrait de Villes d'eaux, Paris : Imprimerie Nationale Editions/Dexia, 2002, p. 51

L'Etat sarde aidé par les communes savoyardes finance les différents projets d'infrastructures comme par exemple en 1856 la construction pour 900000 francs de thermes (fig. $\mathbf{n}^{\circ}$ 4). En 1860, lors du rattachement à la France, l'Etat français fournit 700000 francs pour l'achèvement des thermes en échange du retrait de la ville d'Aix qui partageait avec l'Etat sarde la direction du domaine thermal. Les bénéfices réalisés par l'Etat sont importants à Aix, ce qui justifie son maintien comme gestionnaire et propriétaire. Pourtant la loi de finances de 1884 impose à l'Etat de mettre en ferme les thermes d'Aix-les-Bains, considérant que le gouvernement n'avait pas à gérer de tels établissements, d'autant qu'à la même époque les recettes tendent à diminuer. Mais cette tentative échoue et la régie demeure.

43 A Vichy, comme à Plombières, voire dans une certaine mesure à Aix-les-Bains, rares sont les entrepreneurs, les financiers, les industriels qui prennent à ferme les établissements thermaux. A chaque affermage les demandes sont très restreintes.

A Plombières, l'impulsion donnée par l'empereur est nécessaire ; à Vichy, il n'existe en réalité en 1853 qu'une seule proposition sérieuse qui est soumise à l'approbation de l'administration; à Aix-les-Bains lorsque des tentatives d'affermage sont réalisées, il ne semble pas que des fermiers aient répondu.

L'importance du rôle de Napoléon III est considérable, dans les trois exemples cités, les changements radicaux dans la gestion étant intervenus entre 1853 et 1870.

L'Etat est en mesure, selon le bon vouloir du gouvernement d'apporter des sommes importantes dans la gestion thermale: ce fut le cas à Vichy, à Aix-les-Bains et à Plombières. Néanmoins, il ne peut pas gérer l'ensemble du patrimoine thermal français, il n'a ni les moyens financiers, ni même la volonté d'entreprendre des investissements aussi considérables. La possession par l'Etat donne une image de sérieux à l'exploitation 
thermale, tout comme le fait qu'une eau soit autorisée par l'Etat cautionne son utilisation.

47 A l'inverse, le gage de sérieux peut être à l'origine d'un sentiment d'ennui, de lieu uniquement consacré à la maladie, où les distractions sont absentes. Ce constat est contre-balancé par l'exemple vichyssois.

Des inconvénients existent comme celui d'un cahier des charges très contraignant. La "garantie» de l'Etat n'induit pas une réussite "automatique", même si elle peut s'avérer un facteur non négligeable de succès.

49 A partir de la décennie 1850, on remarque un changement de l'importance des fermiers : à de simples particuliers, issus des environs, voire de la localité thermale, se substituent de puissantes compagnies regroupant des actionnaires d'origines géographiques et sociales beaucoup plus larges que les seuls " artisans " locaux. De plus, la durée de la ferme a évolué : de trois ans, elle passe souvent à plusieurs décennies ; cette augmentation apparait comme une nécessité pour espérer obtenir des sociétés concessionnaires des engagements de construction d'infrastructures thermales et pour permettre une certaine continuité dans le mode de gestion en favorisant la mise en place de politiques d'investissement cohérentes.

Le choix de la régie favorise une gestion moins axée sur la rentabilité tout en accordant une place plus importante à la médecine surtout lorsqu'elle s'adresse aux catégories sociales les plus défavorisées. Dans la gestion des sources thermales par l'Etat se trouve une contradiction essentielle de l'histoire du thermalisme : le souci de faire bénéficier le plus grand nombre des bienfaits des eaux minérales, de diffuser le plus largement possible les pratiques thermales, tout en prenant en compte les soucis de rentabilité. La santé ou l'économie, la santé et l'économie, il s'agit là d'une équation difficile à résoudre, surtout lorsque le propriétaire des sources est l'Etat.

51 Après l'évocation des investissements étatiques et de la gestion par l'Etat d'une partie $\mathrm{du}$ thermalisme, il faut prendre en considération les sources appartenant aux communes et aux départements.

\section{Municipalité et département : gérer la difficulté financière}




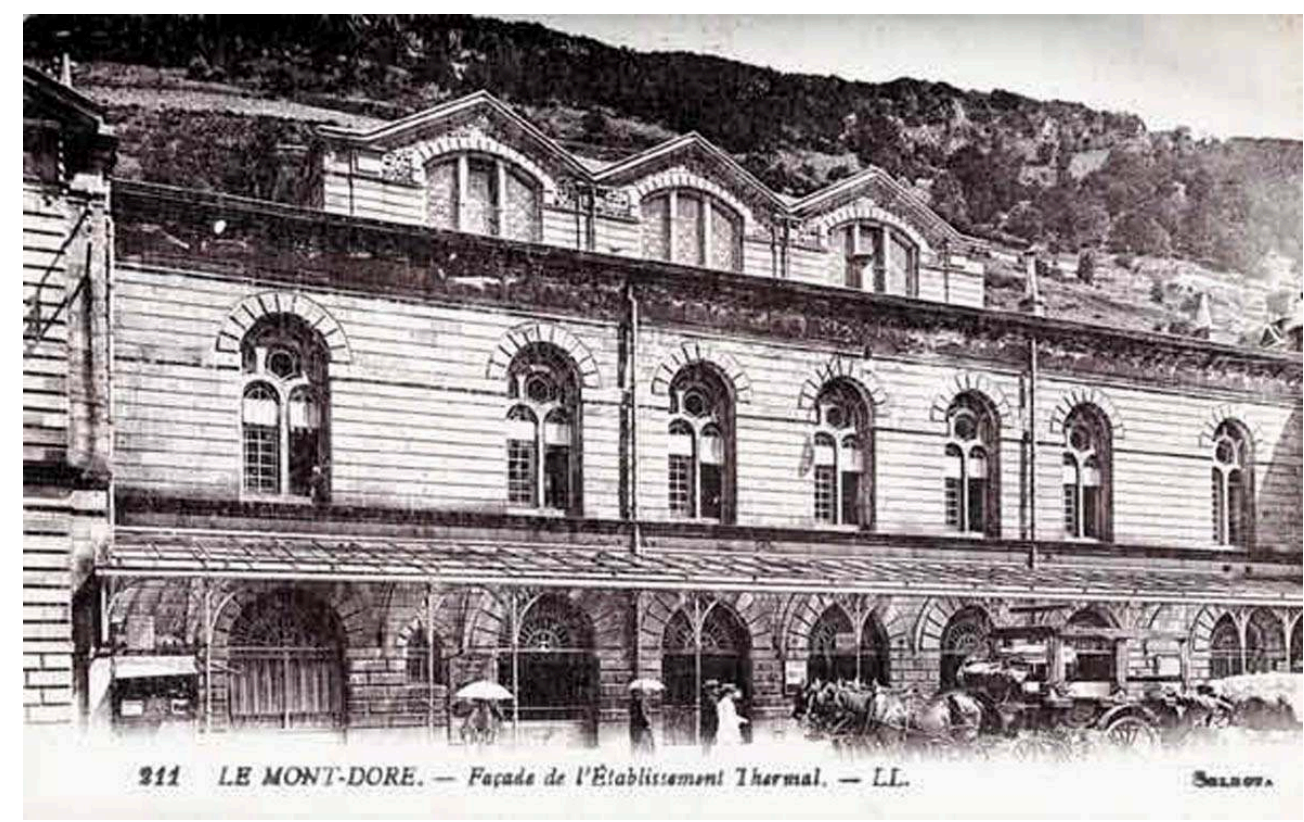

Le Mont-Dore (Puy-de-Dôme). Etablissement thermal, façade antérieure. Conçu en 1887 par I'architecte Emile Camut et achevé en 1894 à partir d'un édifice antérieur. Carte postale, début XXe s., coll. B. Toulier. Extrait de Villes d'eaux, Paris : Imprimerie Nationale Editions/Dexia, 2002, p. 53.

52 Les stations thermales qui possèdent des sources appartenant aux communes sont relativement nombreuses, environ une cinquantaine dont « seulement » une dizaine de renommée nationale (Luchon, Barèges, Cauterets, Royat, Eaux-Bonnes, Bagnères-deBigorre, Saint-Sauveur, Evian, Thonon). Rares sont les sources propriétés des départements : six ou sept pendant tout le XIXe siècle, dont la plus notable est le MontDore dans le Puy-de-Dôme, (fig. $\mathbf{n}^{\circ}$ 5) et quatre en Corse.

Dans ces stations, toutes les sources n'appartiennent pas obligatoirement à la municipalité : à Evian, par exemple, à la fin du XIXe siècle, deux des neuf sources jaillissant sur le territoire de la commune sont propriétés municipales, les autres sont gérées par des investisseurs privés.

De nombreuses communes sont devenues propriétaires des sources thermales à la suite des saisies révolutionnaires. De nombreux points communs existent entre la gestion du thermalisme par l'Etat et par les communes ou les départements. Parmi les ressemblances, on peut relever le mode de gestion qui évolue entre l'affermage et la régie.

55 L'appel à des particuliers ou à des sociétés capitalistes paraît être la solution la plus prometteuse. Le manque d'argent est une constante dans l'histoire du thermalisme des collectivités territoriales. Certaines communes parviennent cependant à effectuer des constructions coûteuses grâce à des emprunts et à des sacrifices importants.

A Luchon, la municipalité dépense 800000 francs pour élever un magnifique monument thermal au milieu du XIXe siècle. Une somme considérable qui permet à la station thermale de réellement lancer le thermalisme local.

57 Les communes connaissent généralement d'importantes difficultés financières dans la gestion des sources et des établissements thermaux, difficultés qui deviennent 
insurmontables lorsque des constructions ou des améliorations importantes aux infrastructures existantes sont nécessaires.

58 A la différence de l'Etat certaines communes ou départements ont dû abandonner la propriété des sources pour tenter de conserver une exploitation thermale sur leur territoire.

Ainsi en 1861, le département de la Lozère vend les sources de La Chaldette à un rentier de Montpellier pour 19000 francs. Bien souvent, lorsque les communes décident de conserver leur domaine thermal, la gestion est chaotique, ballottée entre différents gestionnaires privés dont la plupart ne parviennent pas à assurer financièrement la croissance thermale, ni même parfois à simplement maintenir le statu quo. Comme pour les sources appartenant à l'Etat, on note une évolution au cours du XIXe siècle, avec un affermage en faveur des habitants des environs des griffons dans un premier temps, puis au milieu du XIXe siècle, à des investisseurs souvent plus lointains.

Un nouveau palier est atteint au cours de la décennie 1870, avec la création de sociétés capitalistes regroupant des financiers provenant d'horizons géographiques et professionnels plus larges et qui cherchent dans le thermalisme l'occasion d'effectuer des profits importants. Evidemment, ce schéma d'évolution ne correspond réellement qu'à des stations thermales assez fortunées pour attirer des investisseurs prêts à apporter d'importantes sommes d'argent dans la gestion des ressources thermales municipales.

61 Certaines sources communales se «contentent» d'être gérées par des modestes fermiers incapables financièrement de construire des infrastructures d'exploitation similaires à celles présentes dans les villes d'eaux les plus réputées. C'est en effet une des particularités de l'économie thermale. Elle repose sur une matière première disponible à faible coût, mais doit prendre en compte son image et son environnement pour espérer prospérer, face à une demande volatile qui accorde beaucoup d'attention aux apparences.

Certaines municipalités ne considèrent pas réellement le thermalisme comme une priorité. Dans les esprits des édiles municipaux, il s'agit parfois davantage d'une contrainte que d'une chance. A Aix-en-Provence par exemple, la commune ne pratique pas une gestion dynamique de son potentiel thermal, elle se contente de quelques améliorations lorsque l'exploitation dégage quelques excédents. Cet intérêt limité se manifeste lors de l'affermage de 1859: la commune ne pose aucune condition financière lors de la concession. 




La Bourboule (Puy-de-Dôme). Etablissement thermal, corps central du bâtiment latéral. Par l'architecte départemental Agis-Léon Ledru (1876-1877). Carte postale, début XXe s., coll. B. Toulier. Extrait de Villes d'eaux, Paris : Imprimerie Nationale Editions/Dexia, 2002, p. 52.

Certaines communes qui choisissent l'affermage font un choix judicieux, c'est le cas par exemple pour la station thermale de La Bourboule, lors de l'affermage des sources communales sous le Second Empire à une compagnie qui, en échange participe au développement de la station (participation à la création du chemin de fer, à la construction d'une église, d'un bureau de poste, réalisation de routes...) (fig. $\mathbf{n}^{\circ} \mathbf{6}$ ).

En parallèle au schéma général et pour corroborer l'idée de diversité dans le thermalisme, il existe dès le début du XIXe siècle des exemples de création de sociétés capitalistes qui décident d'investir dans le thermalisme. En 1819, une société capitaliste par actions se met en place pour gérer les sources communales de Brides en Savoie. Son capital social est de 30000 francs. Elle a été créée sous l'impulsion d'un médecin, le docteur Hybord ; elle reçoit le soutien du roi de Piémont-Sardaigne, Victor-Emmanuel, qui prend quatre actions de la société ; la province de la Tarentaise quant à elle investit dans quatorze actions de 500 francs. Les autres actionnaires sont des propriétaires de la province de la Tarentaise. La gestion est bien du ressort d'investisseurs privés mais l'administration n'est pas absente ; lors des conseils d'administration, les représentants de la province sont présents et interviennent fréquemment. Les sacrifices financiers pour la construction d'un nouvel établissement thermal, en dépit de plusieurs augmentations de capital, qui ont d'ailleurs du mal à se réaliser, sont trop importants et la société en moins de deux décennies, en 1833, doit s'avouer vaincue et cède la gestion du patrimoine des établissements thermaux à la province de la Tarentaise.

65 Le thermalisme ne semble pas encore assez mûr pour accueillir réellement le capitalisme dans sa forme la plus aboutie de société par actions. L'incapacité de pouvoir édifier un établissement thermal digne de ce nom faute de capitaux explique en partie cet échec. Après une histoire chaotique de tentatives de gestion départementale ou communale, les sources sont vendues sous la Troisième République à un particulier. 

département ont une plus importante envergure financière et plus d'ambition. Désormais il est mentionné que les fermiers doivent participer à la construction des nouveaux bâtiments, le bail de 1887 prévoit même que la dépense doit être de 1,8 million entre 1888 et 1892 pour construire de nouveaux thermes.

Figure 7

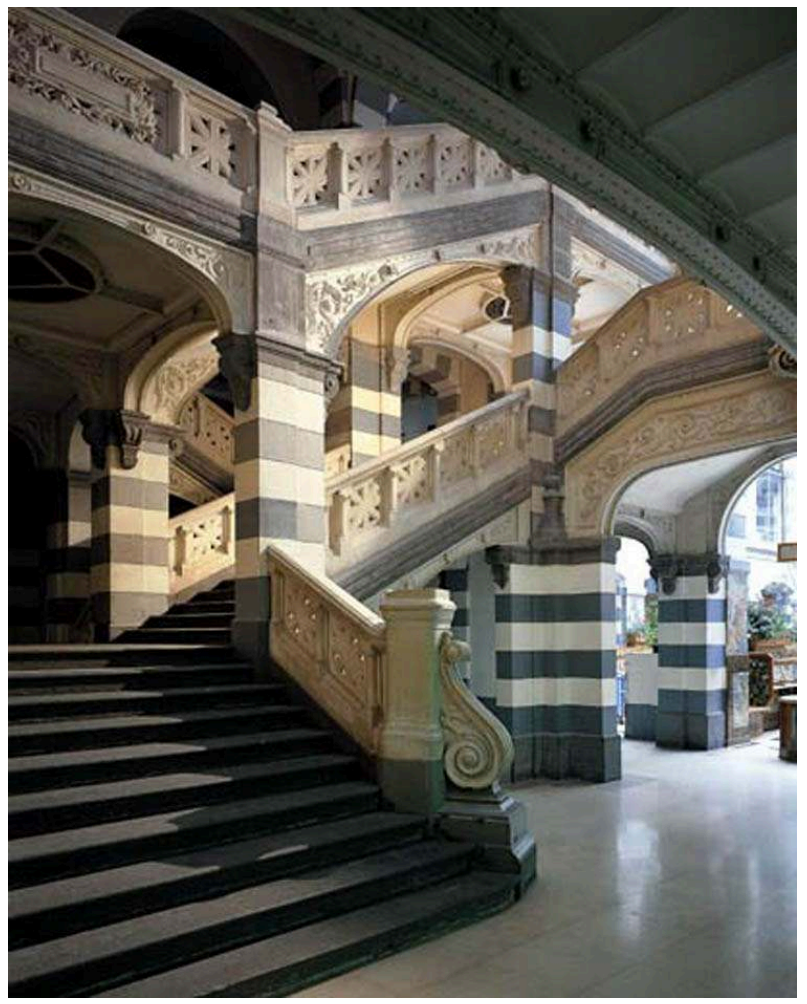

Le Mont-Dore (Puy-de-Dôme). Etablissement thermal, escalier monumental à triple volée. Photo. Caroline Rose. Extrait de Villes d'eaux, Paris : Imprimerie Nationale Editions/Dexia, 2002, p. 60.

Le fermier d'alors est un dénommé Chabaud, habitant du Mont-Dore, dont on sait seulement qu'il était connu comme propriétaire. Chabaud diversifie son activité en 
achetant des hôtels. La concession des thermes s'accompagne de celle des salons et du casino. Chabaud transforme plus tard sa société en société anonyme par actions, dont les actionnaires sont issus du département du Puy-de-Dôme. Le Mont-Dore est une réussite pour le département du Puy-de-Dôme en terme d'image et sur le plan financier (fig. $\mathbf{n}^{\circ}$ 7).

70 Avant de terminer ce tour d'horizon de la situation des thermes publics, évoquons le cas atypique de plusieurs stations pyrénéennes. En effet, plusieurs sources hydrominérales sont gérées non par une seule commune mais par un syndicat de communes, regroupant plusieurs municipalités situées dans une même "vallée ». Il y a donc création d'une structure intermédiaire entre la commune et le département.

71 Le cas le plus abouti est celui de Cauterets : jusqu'en 1840, les possessions indivises des communes de la vallée de Saint-Savin sont en réalité gérées par l'administration préfectorale, au bénéfice des communes. La création du syndicat, en relation avec la loi sur l'administration communale de juillet 1837, permet le transfert de l'administration des thermes directement à la commission syndicale. Pendant la première moitié du XIXe siècle, les sources communales de Cauterets sont affermées à de "petits fermiers " locaux. Certains de ces adjudicataires cumulent les fermes: en 1815, un dénommé Larrieu obtient la gestion des thermes de Cauterets et de Barèges. Les fermiers sont avant tout de petits propriétaires de Cauterets ou de ses environs, qui luttent pour obtenir ces fermes, qui ne génèrent pas obligatoirement des revenus, mais confèrent un certain prestige. Les différentes infrastructures nécessaires sont financées par le syndicat de communes. Le changement a lieu sous le Second Empire: en 1864-1865 est fondée "la Société anonyme des eaux de Cauterets» qui obtient la concession de l'ensemble des sources minérales et des thermes de Cauterets. Le dirigeant de cette société est Benjamin Dulau, entrepreneur de travaux publics domicilié dans les Landes mais travaillant à Paris. Ce sont surtout des Bordelais qui composent la société, majoritairement des entrepreneurs et des négociants ; une fois encore il s'agit davantage de réseaux relationnels d'amis que d'actionnaires d'horizons divers qui se regroupent uniquement pour gérer une entreprise. La société Dulau entreprend une politique d'expansion : création de chalets, d'hôtels, d'un casino.

Les rapports sont souvent houleux entre le concessionnaire et le syndicat de vallée qui ne voit pas d'un très bon œil l'importance grandissante de ces «étrangers ». Lors du renouvellement de la concession, une nouvelle société prend la suite de celle de Dulau. Elle est également composée majoritairement d'entrepreneurs, de propriétaires, qui sont surtout originaires des Basses et des Hautes Pyrénées. Cette société est rapidement au bord de la faillite, provoquée en particulier par la société Dulau qui, bien que n'étant plus concessionnaire du syndicat de vallée avait établi un domaine thermal concurrent.

73 Il faut attendre 1912 pour qu'une nouvelle concession réussisse; il s'agit cette fois de la Société thermale des Pyrénées, créée sous la direction de Jacques Vernes, administrateur d'une importante banque parisienne et associé à la Compagnie du Midi. Cette puissante compagnie parvient à relancer la station de Cauterets.

74 L'exemple de la gestion publique du thermalisme par l'Etat ou les communautés territoriales montre qu'il faut attendre le milieu du XIXe siècle pour que se mettent en place des sociétés capitalistes qui prennent en charge l'ensemble de l'exploitation thermale dans des stations où les sources sont publiques. Pendant le premier XIXe siècle, l'administration publique s'occupe de la construction, de l'amélioration, de la 
restauration des infrastructures thermales, les investisseurs privés se contentent d'affermer les domaines publics sans réellement modifier les infrastructures.

Les premiers fermiers des domaines thermaux publics sont essentiellement des habitants de la région. La population locale s'intéresse donc de près à la gestion des ressources hydrominérales de sa commune.

Dans la seconde partie du siècle, les investisseurs privés se manifestent et mettent en valeur de nombreuses stations " publiques».

Le Second Empire a souvent constitué un tournant, au moment même où l'avenir du thermalisme et sa rentabilité semblent assurés, où le chef de l'Etat a un intérêt personnel pour les eaux et surtout, où le capitalisme s'impose et permet de réunir des capitaux suffisants pour subvenir aux énormes besoins nécessités par le thermalisme.

Il faut pourtant demeurer prudent, s'il semble que les stations thermales soient des lieux propices à l'intervention capitaliste, toutes n'ont pas attiré de l'argent en quantité suffisante. De plus, certaines sources thermales gérées directement par l'Etat prospèrent, même si ce dernier cas demeure exceptionnel.

\section{Capitalisme, médecine : la croissance du secteur privé dans le thermalisme}

79 La gestion privée est omniprésente dans le thermalisme, d'une part parce que la plupart des sources appartiennent à des particuliers, à des sociétés privées, et d'autre part parce qu'à de multiples reprises et près de nombreux griffons, l'Etat, les départements, les communes font appel à des investisseurs privés.

80 A plusieurs reprises à partir du milieu du XIXe siècle, il apparaît que les sociétés capitalistes sont l'expression de l'intérêt général pour le thermalisme et n'apparaissent plus uniquement comme des entreprises ayant pour seul but de faire des bénéfices; elles deviennent les garantes de la prospérité locale. Le changement est important par rapport à la situation antérieure, lorsque les entreprises capitalistes apparaissaient comme les « diables » venus dépouiller les habitants de leurs biens.

81 Tout au long du XIXe siècle, on relève des initiatives d'individus "solitaires", qui croient dans le pouvoir des eaux et pensent qu'ils peuvent devenir des « alchimistes du thermalisme "; ils se lancent alors dans l'exploitation thermale : le succès peut être au bout de la ligne droite, mais l'échec n'est pas exclu. Les réussites peuvent être éclatantes, des fortunes sont nées grâce à l'eau minérale : Louis Bouloumié à Vittel, le docteur Vidart à Divonne. Celle d'Auguste Badoit, à Saint-Galmier est exemplaire : ancien "voyageur de commerce en soierie", il crée à Saint-Galmier une entreprise prospère basée essentiellement sur l'expédition d'eau minérale. Sa réussite est en partie due aux qualités des eaux de Saint-Galmier qui supportent très bien le transport en bouteilles, mais surtout à la clairvoyance de Badoit qui comprend rapidement que le thermalisme près des sources n'est pas la meilleure solution pour faire fortune à SaintGalmier et qu'une publicité importante peut seule permettre une croissance des ventes. 
Figure 8

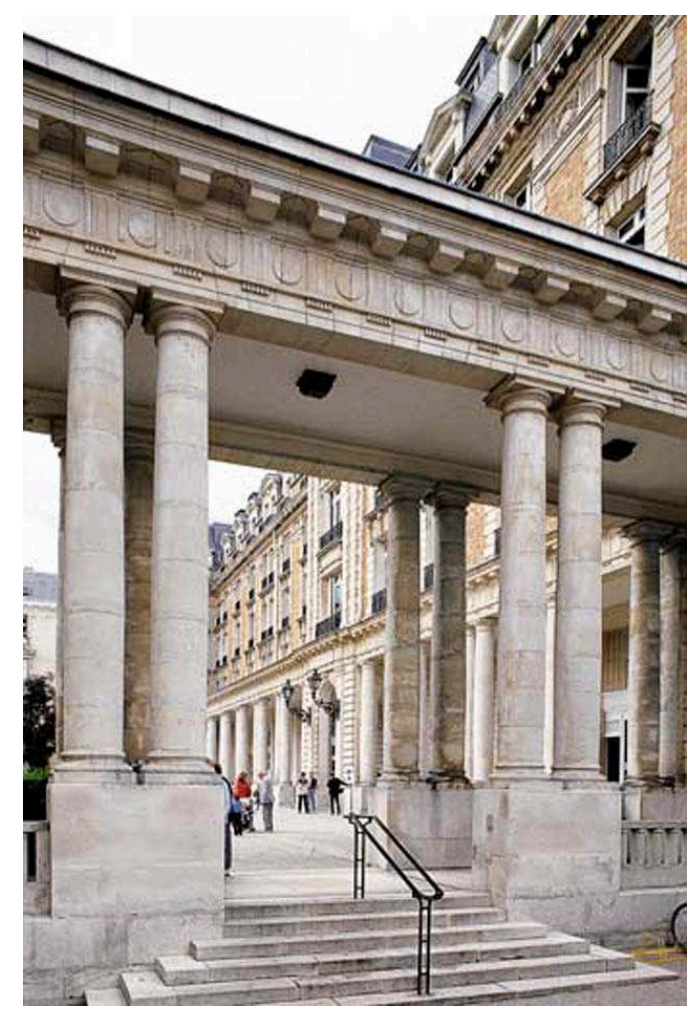

Vittel (Vosges). Le Grand-Hôtel, extérieur. Reconstruction par Georges Walwein en 1912. Photo. Caroline Rose. Extrait de Villes d'eaux, Paris : Imprimerie Nationale Editions/Dexia, 2002, p. 141.

82 Mais c'est aussi l'exemple d'un avocat de Rodez, Louis Bouloumié, contraint à l'exil politique et qui en 1852 décide après une cure thermale dans la station voisine de Contrexéville de lancer la station thermale de Vittel (fig. $\mathbf{n}^{\circ} \mathbf{8}$ ). Louis Bouloumié, tout comme Auguste Badoit, débute son exploitation grâce à des fonds personnels, la croissance est lente mais sûre. Dans les deux cas l'auto-financement est la règle, l'appel à des emprunts est effectué en dernier recours. La volonté de conserver la mainmise sur l'ensemble de la gestion explique l'absence d'autres investisseurs.

Ce n'est que durant la décennie 1880 que les Bouloumié doivent se résoudre à créer une société d'actionnaires, la «société de Vittel ", au capital de 1,7 millions de francs. La famille Bouloumié conserve la tête de l'entreprise, les autres actionnaires sont surtout M. Koechlin-Schwartz, manufacturier, maire du 8e arrondissement de Paris, un banquier des Vosges, Jules Evrard, un maître de forges, Henri de Tricornot, en fait un mélange d'investisseurs locaux et parisiens, faisant tous partie des relations personnelles de la famille Bouloumié. 


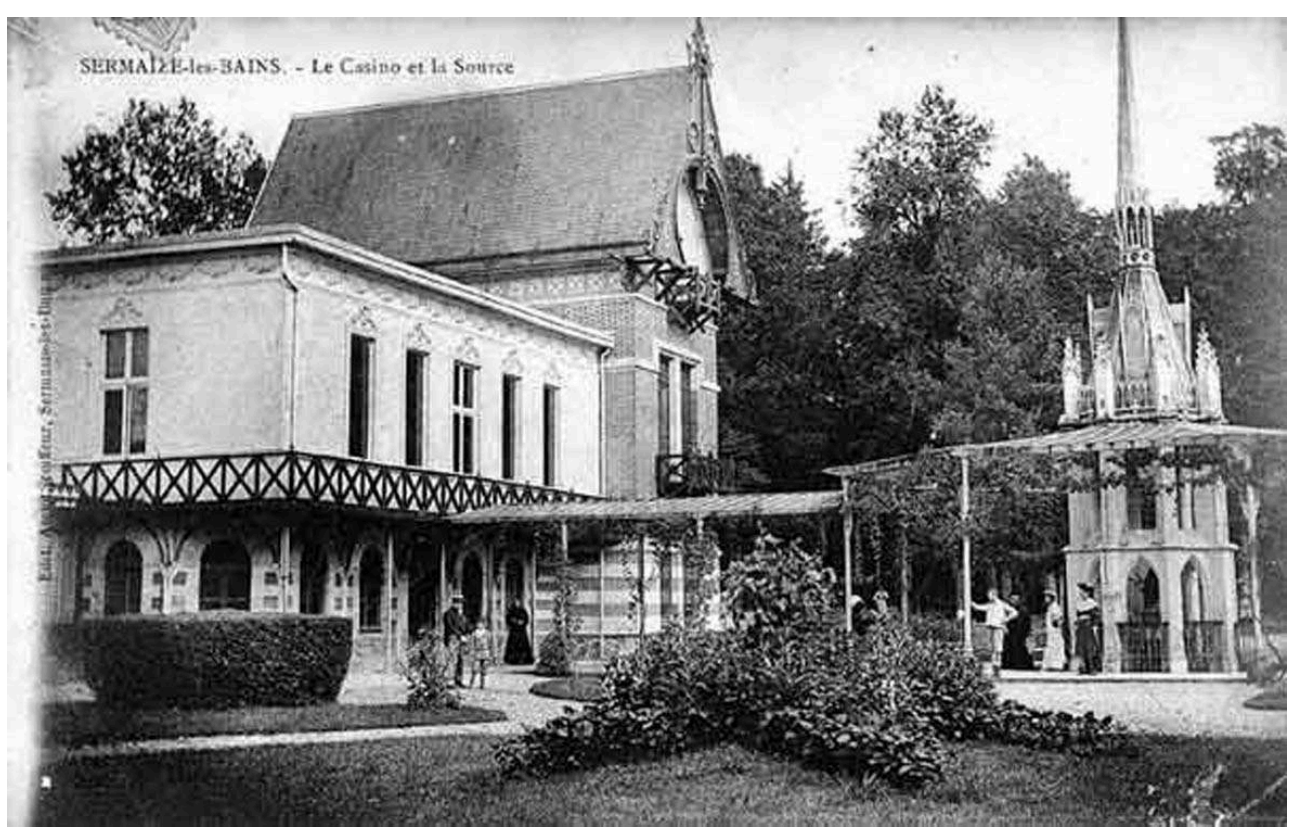

Sermaize-les-Bains (Marne). Casino et buvette construite par la Compagnie des Eaux par l'architecte Grandidier vers 1852. En 1895, agrandissement du casino et premier étage converti en salle de spectacle. Carte postale, début XXe s., coll. B. Toulier. Extrait de Villes d'eaux, Paris : Imprimerie Nationale Editions/Dexia, 2002, p. 71.

La domination de la famille Bouloumié perdure bien au-delà de la Première Guerre mondiale, sans aucune remise en cause. Les exemples évoqués sont des destins toutefois exceptionnels dans le thermalisme. Pendant la première partie du XIXe siècle, la majorité des propriétaires de sources se contente de gérer sans grande ambition les griffons, réduisant au minimum les investissements. Pourtant dès le début du XIXe siècle, les tentatives de particuliers pour lancer des stations thermales sont nombreuses. Ce sont souvent des médecins, des avocats qui tentent leur chance, mais aussi de petits propriétaires, des artisans qui essaient de développer l'exploitation de sources hydrominérales, ce que l'on peut appeler un «artisanat thermal », qui tend à disparaître peu à peu au cours du XIXe siècle, tandis que les médecins, les hommes de loi, les architectes continuent à jouer un rôle prépondérant soit en tant que propriétaires et initiateurs uniques de la gestion thermale, soit en participant aux nombreuses sociétés thermales qui se créent (fig. $\mathbf{n}^{\circ} \mathbf{9}$ ).

La « fièvre thermale » est une expression littéraire qui correspond à l'augmentation du nombre de personnes se rendant chaque année près des sources et caractérise également l'augmentation $\mathrm{du}$ nombre de sources en exploitation. Elle permet également d'identifier l'importance de la «ruée économique et financière » vers le thermalisme, l'espoir d'enrichissement, la recherche du profit qui expliquent le nombre considérable de créations d'exploitations thermales et le nombre important de forages pour découvrir des sources. A Vals par exemple en 1870, 80 sources sont exploitées par plus de trente propriétaires.

86 La croissance du nombre de sources exploitées en France va de pair avec une augmentation du nombre de propriétaires, ce qui indique que la volonté de gagner de l'argent, la recherche de nouvelles émergences de l'eau minérale correspond bien à un 
projet « industriel» et mercantile, loin des découvertes fortuites du début du XIXe siècle.

87 Loin des fastes des grandes stations nécessitant d'importants investissements, des exploitations thermales « artisanales » souvent familiales parviennent à se maintenir pendant une grande partie du XIXe siècle, citons le cas des établissements thermaux de Pont-les-Bains, trois thermes appartenant à trois familles, qui gèrent leur bien jusqu'au début du XXe siècle sans expansion, mais aussi sans faillite.

La fièvre thermale du second XIXe siècle ne concerne pas uniquement les tentatives isolées, elle explique aussi le nombre important de sociétés "capitalistes » créées pour exploiter les loisirs ou les eaux minérales dans les villes d'eaux. En plus des cas précédemment évoqués dans les villes d'eaux où les sources sont publiques, citons l'exemple de Bagnoles-de-l'Orne. En 1865 un notaire d'Alençon, Richard, crée la Société des eaux minéro-thermales de Bagnoles. La réussite n'est pas au rendez-vous; une nouvelle société est constituée en 1880, puis encore une autre en 1896 à l'instigation d'un industriel, Georges Hartog. Cette succession rapide de sociétés n'est pas une exception. A Enghien, les sociétés capitalistes se suivent sans parvenir au succès : en 1863 est fondée sous l'égide d'Albert de Montry la Société des eaux minérales d'Enghien, entreprise de courte durée. En 1868 elle vend ses possessions à une Société des thermes, qui elle-même cède ses propriétés en 1875 à la Compagnie anonyme des eaux d'Enghien-les-Bains, créée par de Villemessant, fondateur du journal Le Figaro. La société végète, et en 1895 une nouvelle entreprise est fondée qui doit à son tour vendre son domaine thermal en 1902 au banquier parisien Bressous.

La fièvre thermale n'a pas seulement concerné de petits villages, de modestes bourgs, la capitale a été aussi le théâtre de création de sociétés capitalistes. En 1874 est créée la Société anonyme des eaux minérales sulfureuses de Belleville-Paris, au capital de 1,6 million de francs. Les fondateurs sont quatre négociants parisiens appartenant vraisemblablement à deux familles, les Lapostolet et les Certeux, mais cette entreprise est sans avenir. La litanie des échecs et des successions de sociétés capitalistes pourrait encore être longue, preuve à la fois de l'intérêt pour la gestion thermale et de la difficulté d'administrer une exploitation d'eaux minérales. 


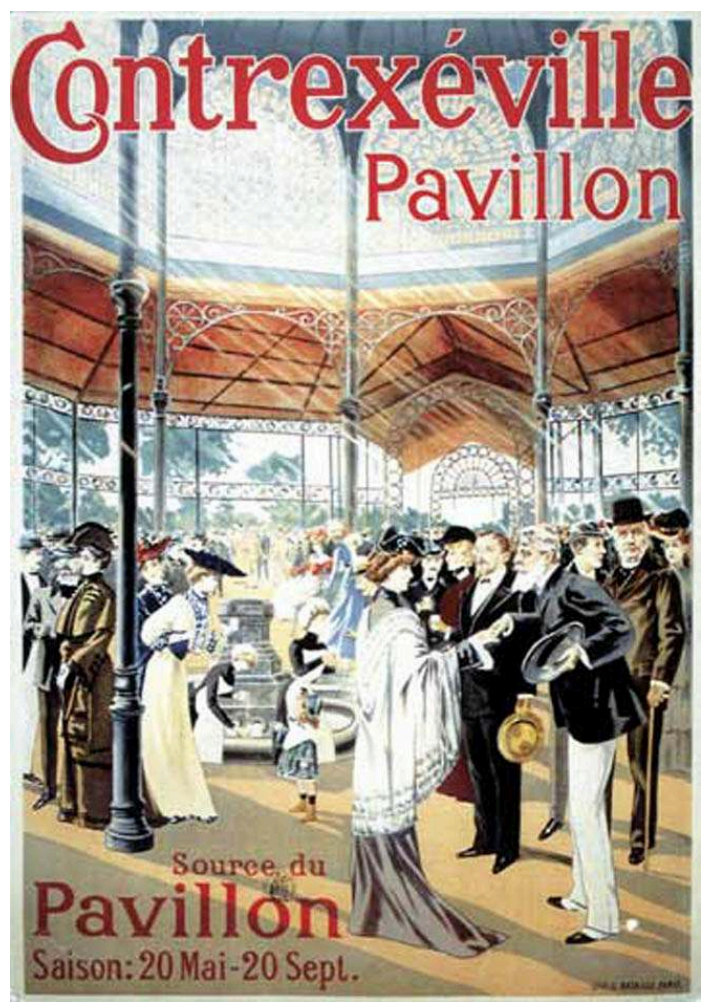

Contrexéville (Vosges). Kiosque à charpente métallique sur plan octogonal, buvette édifiée en 1880, intérieur. Affiche fin XIXe- début XXe siècle. Repro. Coll. B. Toulier. Extrait de Villes d'eaux, Paris : Imprimerie Nationale Editions/Dexia, 2002, p. 77.

Au début du XXe siècle se créent de puissantes compagnies qui exploitent des sources dans plusieurs stations, avec souvent comme priorité l'embouteillage de l'eau minérale. Citons à titre d'exemple la Compagnie générale d'eaux minérales et des bains de mer, société anonyme au capital de 4,8 millions de francs qui possède des sources à Alet, Allevard, Andabre, Châteldon, Contrexéville, (fig. $\mathbf{n}^{\circ}$ 10) Desaignes, Euzet, Fumades, Salins-du-Jura, Saint-Gervais, Spa, Vals et Vichy. A cette longue liste s'ajoutent des hôtels à Vichy, un casino à Trouville et à Vichy. Cette société est une émanation de la Compagnie fermière de l'établissement thermal de Vichy, et pendant de longues années elle est dirigée par les mêmes personnes.

91 Les différentes sociétés qui se créent peuvent se regrouper en plusieurs catégories en fonction de l'origine de leur investissement. Il y a d'abord celles composées d'acteurs issus des environs des sources, qui cherchent à exploiter au maximum le patrimoine local. Ce type d'entreprises est en règle générale le plus ancien. L'exemple de Bridesles-Bains, dans le premier quart du XIXe siècle, représente bien ce mode d'investissement industriel.

Peu à peu se mélangent les capitaux locaux et les investissements provenant des grandes métropoles, qui trouvent dans les stations thermales des terrains propices à l'extension de leur activité. Dans ces sociétés, les banquiers jouent un rôle de premier plan.

93 A ces banquiers se joignent des industriels tels que des entrepreneurs de travaux publics, souvent en contrat avec l'Etat pour des marchés publics, mais aussi des médecins, qui faute d'apports importants en capitaux apportent leurs savoir-faire, 
leurs compétences, leur présence, qui est un gage de sérieux pour l'image de l'entreprise.

94 L'association entre la banque et les entrepreneurs de travaux publics se rencontre notamment à Vichy au sein de la Compagnie fermière.

A sa fondation les deux principaux acteurs de cette société sont deux industriels qui ont fait fortune, Antoine Callou et Auguste Lebobe. A ces deux acteurs principaux s'est joint Denière, régent de la Banque industrielle et de la Banque de France de 1866 à 1888 et qui joue un rôle actif dans l'évolution de la Compagnie. Il s'occupe d'acquérir les sources concurrentes, participe à la création d'une société « parallèle » à la Compagnie fermière, chargée de gérer des activités thermales qui ne peuvent pas être comprises dans la Compagnie fermière, notamment les jeux d'argent, avant que l'autorisation ne soit donnée par l'Etat de pouvoir pratiquer les jeux de hasard dans son casino.

Il n'est pas exceptionnel que des membres de la haute banque parisienne participent à des opérations financières dans le thermalisme. Cette participation devient plus importante à la fin du XIXe siècle et au début du XXe siècle. Isaac Péreire investit d'importants capitaux pour acquérir des sources minérales dans la station thermale d'Amélie-les-Bains en 1863. Malheureusement pour la ville d'eaux pyrénéenne, les déboires financiers de la famille Péreire à partir de 1867 réduisent les investissements effectués dans le thermalisme. Néanmoins lors des premières années de sa gestion, Isaac Péreire a modernisé profondément l'établissement thermal, des promenades ont été aménagées, des chalets ont été construits. L'achat des thermes d'Amélie participe à la politique industrielle d'Isaac Péreire dans le Sud-Ouest, politique qui trouve son point d'orgue dans la direction de la Compagnie des chemins de fer du Midi. Après la mort du banquier en 1880, les thermes passent sous le contrôle d'une société créée par ses héritiers, la Société Péreire, qui poursuit la gestion du domaine thermal sans pourtant amener une croissance de la fréquentation.

Notons aussi le rôle de Jacques Vernes qui crée à partir de Cauterets la puissante Société thermale des Pyrénées et qui participe aussi à la Compagnie du Midi, dans une alliance qui a pour but de rentabiliser les chemins de fer et de faciliter l'accès aux stations thermales pyrénéennes souvent enclavées. 
Figure 11

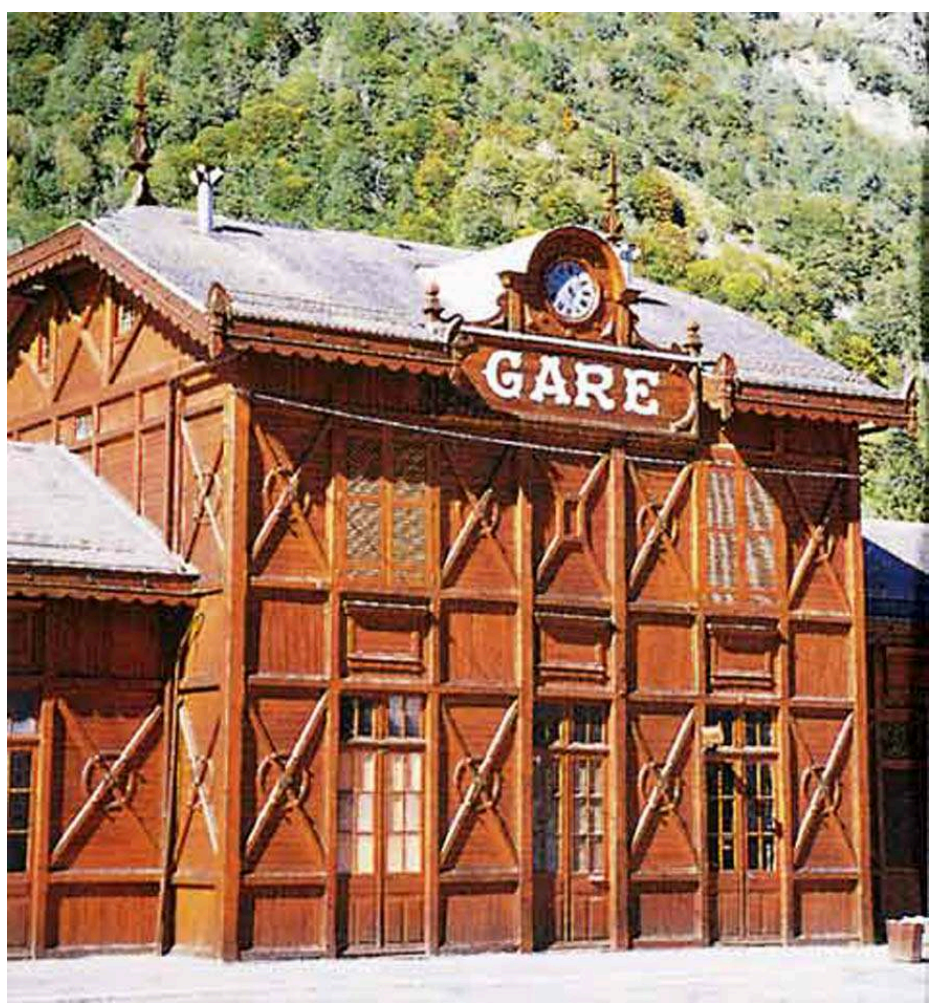

Cauterets (Hautes-Pyrénées). Gare ferroviaire pour la Compagnie du Midi. Edifiée par l'entreprise Médevielle sous la direction de Ferrier, ingénieur de la Compagnie. Repro. Extrait de Villes d'eaux, Paris : Imprimerie Nationale Editions/Dexia, 2002, p. 20.

Dans un article consacré aux liens entre le thermalisme pyrénéen et la Compagnie du Chemin de fer du midi, Christophe Bouneau met en lumière l'importance des villes d'eaux pyrénéennes dans la constitution d'un réseau ferré montagnard, situation qui est proche de ce qui a lieu dans les autres massifs montagnards français (fig. $\mathbf{n}^{\mathbf{0}} \mathbf{1 1}^{3}$. Il existe une synergie forte entre le développement du chemin de fer dans les Pyrénées et la croissance du thermalisme.

Sur de nombreuses portions du réseau ferré, ce sont les stations thermales qui justifient la présence de la plupart des voyageurs; réciproquement, les villes d'eaux bénéficient de moyens de communication pour drainer la clientèle. La Compagnie du chemin de fer du Midi adapte son offre à la demande thermale en proposant des réductions spéciales pour les séjours thermaux et en construisant des tronçons de voies ferrées qui servent presque uniquement aux curistes. En 1913, les administrateurs de la Compagnie du Midi peuvent déclarer avec satisfaction : « la fréquentation croissante de nos stations thermales et balnéaires atteste le succès de nos efforts multiples pour adapter toujours mieux nos services aux besoins du public ».

Le chemin de fer est considéré comme l'élément clé de la Révolution industrielle en France, un facteur de développement du thermalisme: si le train ne génère pas obligatoirement une extension du bassin de recrutement de la demande, il est toutefois à l'origine d'une intensification des flux lointains, grâce au raccourcissement des temps de trajet. A Contrexéville, c'est Achille Fould qui a pris part à la constitution d'une société qui en 1864 se porte acquéreur de la source des Pavillons : la Société des eaux de Contrexéville. La famille Fould participe à la gestion de la station vosgienne jusqu'à la 
Première Guerre mondiale. Cette entreprise est une réussite et contribue au développement de la station par la construction d'un " nouveau Contrexéville ».

101 A Evian, après une tentative peu fructueuse initiée à partir de 1826 par le genevois Fauconnet, qui parvient à construire un hôtel mais qui n'a pas suffisamment de moyens financiers pour améliorer les thermes, une société de sept actionnaires qui prend le nom de Société anonyme des eaux minérales d'Evian est créée en dépit de ce nom " pompeux », elle ne possède qu'une source à Evian. Cette société se modifie à plusieurs reprises : à la fin du Second Empire, son emprise est presque totale sur la station, elle a acheté la plupart des sources concurrentes, et a construit un hôtel monumental.

Le principal instigateur de la croissance d'Evian et de l'entreprise est Alfred André qui fait appel au soutien de la haute banque protestante pour investir à Evian et dans la Société des eaux. Lorsqu'il meurt en 1896, c'est son neveu le baron de Neuflize qui prend la direction de l'entreprise.

Les statuts des sociétés qui sont créées tout au long du XIXe siècle pour gérer le thermalisme sont souvent réalisés selon le même modèle, seul change le montant du capital social. Celui-ci peut être relativement limité, par exemple 120000 francs en 1902 pour la Société d'exploitation des eaux et des thermes de Néris jusqu'à atteindre plusieurs millions, dix millions pour la Compagnie nouvelle des Thermes de Cauterets et de la vallée de Saint-Savin en 1895.

Figure 12

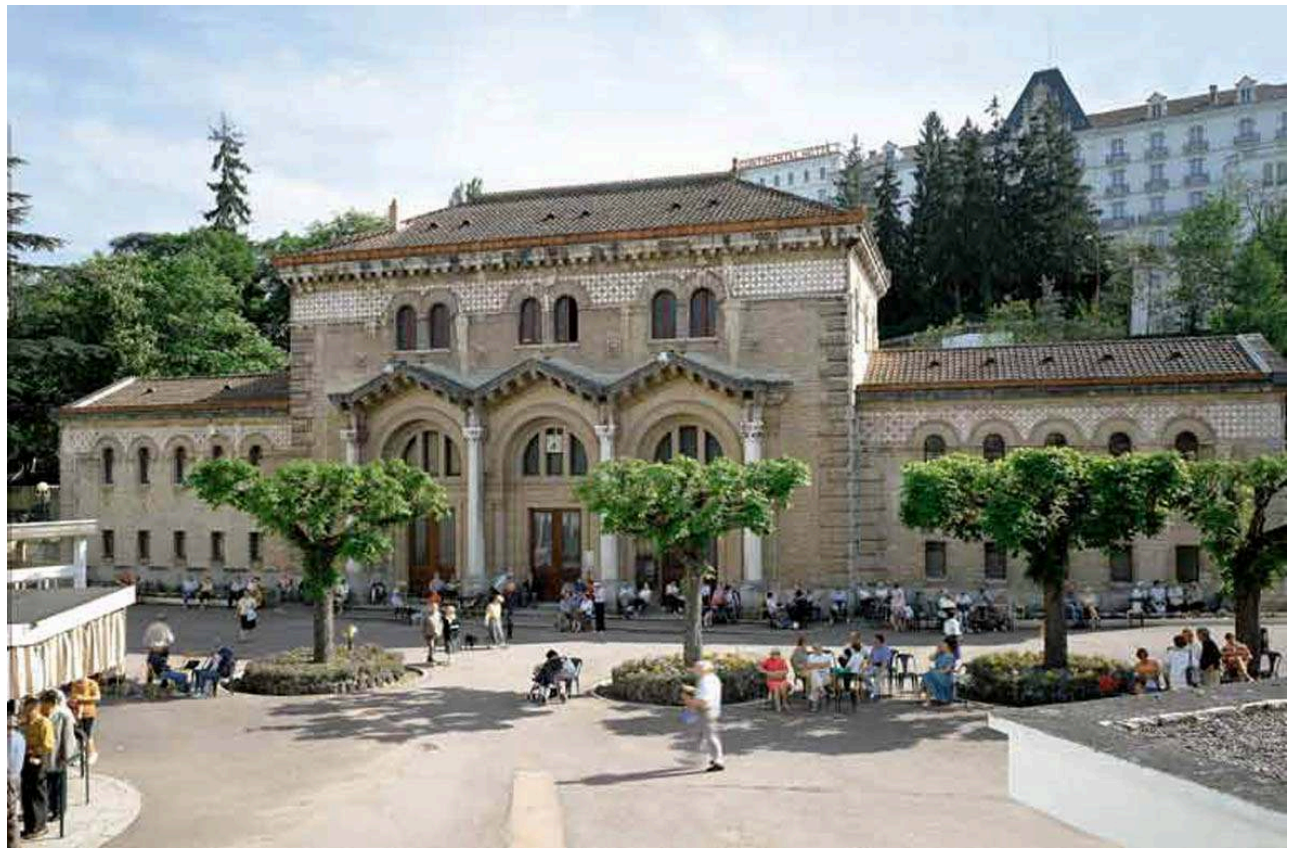

Châtelguyon (Puy-de-Dôme). Etablissement thermal des Grands Thermes, façade antérieure. Par l'architecte Benjamin Chaussemiche en 1906. Photo. Caroline Rose. Extrait de Villes d'eaux, Paris: Imprimerie Nationale Editions/Dexia, 2002, p. 56-57.

Dans les créations de sociétés on retrouve souvent une alliance, presque naturelle dans l'investissement thermal, celle de la banque et de la médecine. Pour illustrer cette association, on peut évoquer la station thermale de Châtel-Guyon dans le Puy-de-Dôme, exemple rare de conglomérat (fig. $\mathbf{n}^{\circ} \mathbf{1 2}$ ). Dans cette station qui végète jusque dans le dernier quart du XIXe siècle, un médecin thermal, le docteur Baraduc, parvient à 
intéresser aux sources châtel-guyonnaises un banquier parisien, François Brocard. Ces deux hommes dirigent totalement l'évolution de la Société des eaux minérales de Châtel-Guyon, qu'ils fondent en 1878 avec des associés dont la plupart ont été contactés par le banquier parisien. Cette association médecine et banque se poursuit après la mort des deux fondateurs, puisque leurs successeurs sont issus de ces deux professions, que ce soient les docteurs Angelby et Pessez ou le banquier Emile Dussargues de Colombier. Le banquier Brocard fondateur de la société de Châtel-Guyon dirige également la Compagnie des eaux minérales de La Bourboule et celle de Royat, trois stations qui sont les principales villes d'eaux du Puy-de-Dôme et parmi les premières de France.

Les sociétés dominées par le banquier Brocard possèdent d'autres investisseurs communs : tous les membres du conseil d'administration de la Compagnie générale des eaux minérales de Royat fondée en 1876 sont liés à François Brocard: le comte de Pontgibaud, un conseiller honoraire à la Cour des comptes, un ancien élève de l'Ecole polytechnique, un comte (conseiller honoraire à la Cour d'appel de Paris), un ingénieur en chef honoraire des manufactures de l'Etat, un libraire. Ces deux derniers administrateurs participent aussi à la Société des eaux minérales de Châtel-Guyon. Les personnalités réunies autour de François Brocard, outre leur apport financier, contribuent par leurs compétences personnelles à la réussite de l'entreprise, par exemple le libraire-éditeur Germer Baillière publie les guides touristiques pour ces stations et des plaquettes publicitaires.

A La Bourboule, on retrouve les mêmes personnes, avec un représentant d'une banque locale : la banque Chalus et frères de Clermont-Ferrand. Ainsi se mêlent intérêts locaux et intérêts parisiens.

Il faut s'arrêter sur François Brocard qui a été à l'origine pour une grande part de la réussite du thermalisme auvergnat de la fin du XIXe siècle. Né à Aumont (Jura) le 9 novembre 1830 , ses parents sont quincailliers. On ne sait rien avant 1860 , date à laquelle selon ses propres écrits, que ne corrobore aucun document, il crée une banque à Paris. La banque Brocard est constituée en société par actions au capital social de 2 millions de francs. Le choix de faire appel à des actionnaires est sans doute imputable aux faibles ressources personnelles de son fondateur, et à la possibilité de compter sur le soutien de ses actionnaires. Quant au capital social, son montant n'a que peu de signification, puisqu'il ne reflète ni les ressources propres $d u$ fondateur, ni les ressources empruntées. La banque Brocard est une banque industrielle; elle évolue aussi dans le monde de la bourse, ce qui explique en partie la fondation par François Brocard du journal «La Rente »4. Le rêve de réunion de différentes stations thermales auvergnates sous la même gestion prend fin avec sa mort en 1897. François Brocard, banquier parisien, a su intéresser au thermalisme auvergnat des investisseurs issus de plusieurs horizons professionnels et géographiques. Le capitalisme auvergnat bien que présent demeure souvent minoritaire.

\section{Le mirage de l'Eldorado}

Il paraît intéressant de s'attarder sur les motivations des investisseurs dans le thermalisme et sur cet eldorado que représentent bien souvent les stations thermales dans leur esprit. Si la constitution de puissantes sociétés capitalistes, les sociétés par actions, parait former le stade ultime et idéal de la gestion thermale, ce constat est loin 
de se vérifier auprès de tous les griffons ; les exemples sont nombreux d'échecs patents malgré la présence d'un important regroupement financier.

En dépit de ces revers, le thermalisme apparaît comme un eldorado, une "terre promise » où l'échec paraît impossible et où l'eau minérale se transforme en or à coup sûr. Il ne faut pas uniquement limiter l'intérêt du capitalisme pour le thermalisme à la seule recherche du profit, le capitalisme pouvait aussi d'une certaine façon rechercher une légitimité, démontrer sa capacité à s'occuper de santé publique et parfois plus efficacement que les pouvoirs publics. Le capitalisme peut ainsi pallier les manques ou en tout état de cause les insuffisances des communes et de l'Etat, même en matière de santé publique.

110 L'argument avancé par certaines sociétés des eaux pour justifier leur présence dans la gestion du thermalisme d'être des garantes de l'eau médicale et plus largement de la santé de la population est mis en avant par les sociétés qui gèrent l'eau potable dans les grandes villes: la première grande entreprise de gestion de l'eau urbaine, la "Compagnie générale des eaux » fondée en 1853 annonce lors de sa création qu'elle ne cherche pas le profit mais qu'elle veut avant tout rendre service à la société et rendre la vie urbaine plus salubre.

Les différents types de gestion favorisent l'existence d'un thermalisme à plusieurs vitesses, une diversité que l'on constate surtout à la fin du XIXe siècle : des sources thermales demeurent gérées comme elles le sont depuis le début du XIXe siècle, d'autres, bénéficiant d'investissements financiers considérables, sont capables de créer toutes les infrastructures nécessaires et éventuellement de résister à des fluctuations de fréquentation.

112 En dépit d'une domination du "privé » sur le «public», du moins si l'on retient le critère de la nature du propriétaire des sources, il semble que les principales stations thermales françaises du XIXe siècle (Vichy, Luchon, le Mont-Dore, La Bourboule, Cauterets, Bagnères-de-Bigorre...) ont bénéficié d'une gestion mixte du thermalisme, c'est-à-dire une possession des sources par l'Etat ou des communautés territoriales et l'intervention de particuliers ou de sociétés « capitalistes » venus apporter des capitaux dans un souci de rentabilité, tout en étant fortement limités dans leur action par le contrôle des propriétaires publics.

113 Au fil du XIXe siècle se profile une évolution dans l'administration des sources, avec une montée en puissance des capitaux nécessaires, accompagnée d'une croissance des associations pour gérer les sources.

114 Peu à peu, de structures familiales ou individuelles demandant peu de capitaux, l'économie thermale évolue vers des structures plus «lourdes ", qui reposent sur des investissements croissants, ce qui n'exclut pas le maintien parallèle de certaines organisations "archaïques » d'administration thermale. L'économie thermale s'avère parfois peu rentable, les réussites sont peu nombreuses, les succès individuels encore plus rares, et pourtant ils servent d'étendard et de faire-valoir. La bonne volonté ne suffit pas à créer une entreprise thermale viable et surtout capable de rivaliser avec les stations thermales les plus importantes. Néanmoins, tout au long du XIXe siècle des sources parviennent au succès : le marché thermal n'est pas sclérosé.

115 Le thermalisme, à quelques exceptions près, n'a pas permis l'enrichissement de ses gestionnaires, tout au plus la confirmation de fortunes déjà établies. Investir dans l'eau 
minérale peut être un moyen d'investissement pour des personnes déjà en possession d'un patrimoine et de revenus importants.

Figure 13

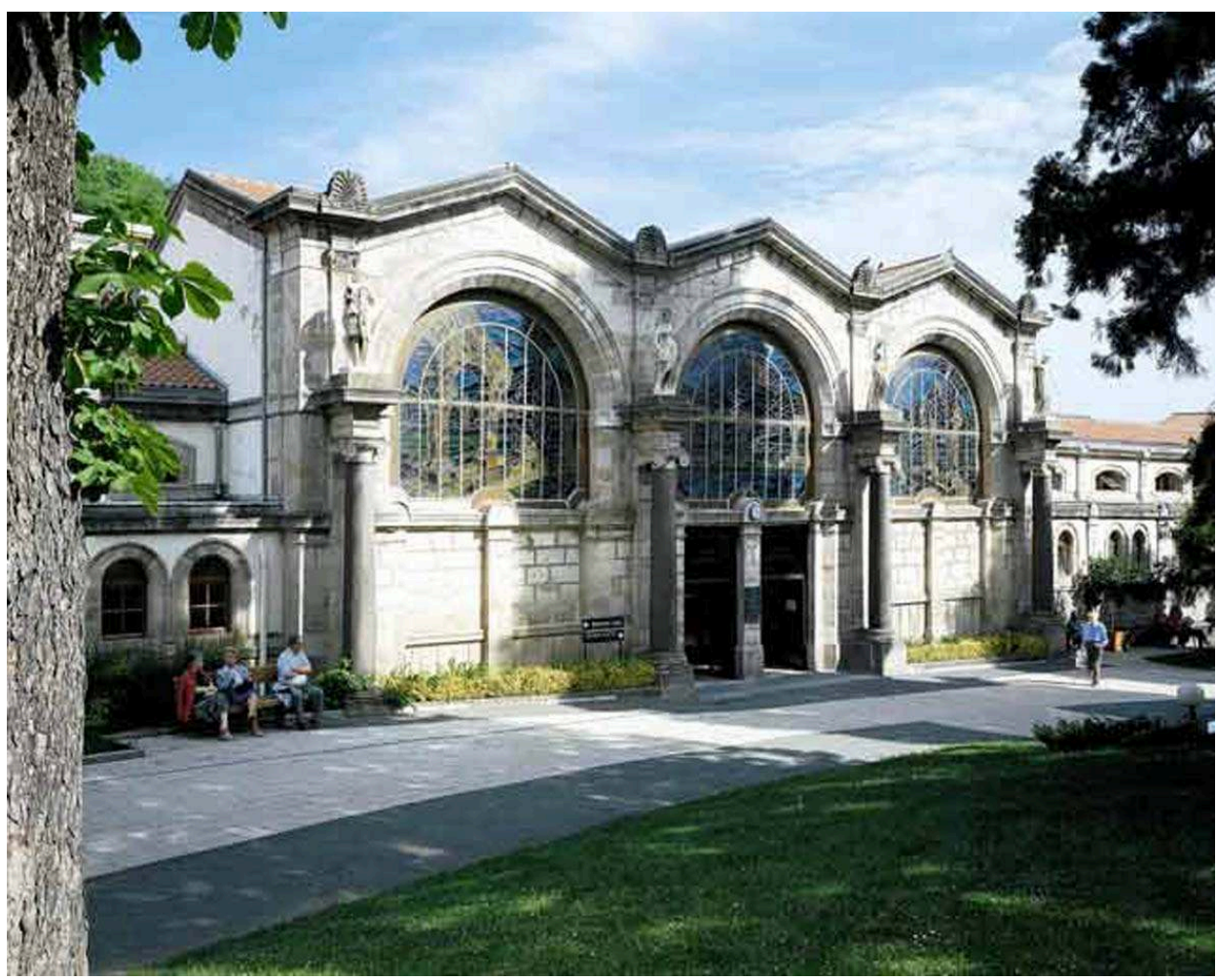

Royat (Puy-de-Dôme). Etablissement thermal, façade antérieure. Par l'architecte Agis-Léon Ledru de 1852 à 1856. Photo. Caroline Rose. Extrait de Villes d'eaux, Paris : Imprimerie Nationale Editions/Dexia, 2002, p. 48-49.

Le thermalisme, du moins dans la gestion des eaux minérales, n'a pas été un réel facteur d'ascension sociale, même si être gestionnaire ou propriétaire d'une source d'eau minérale pendant une grande partie du XIXe siècle permet de jouir d'une position privilégiée au sein des sociétés locales, notamment en tant que vecteur économique et social pour le développement futur du village ou de la ville.

117 Après avoir débuté par une citation de Jules Romains, concluons par une citation de Guy de Maupassant lorsqu'il décrit dans son roman Mont-Oriol l'enthousiasme du banquier Andermatt promoteur d'une nouvelle station thermale: «Je vais probablement tenter une grosse affaire: une ville d'eaux. Je veux lancer une ville d'eaux... Le grand combat aujourd'hui c'est avec l'argent qu'on le livre... Nous sommes les puissants d'aujourd'hui, voilà, les seuls vrais, les seuls puissants.

118 Tenez regardez ce village, ce pauvre village. J'en ferai une ville, moi, une ville blanche, pleine de grands hôtels qui seront pleins de monde, avec des ascenseurs, des domestiques, des voitures, une foule de riches servie par une foule de pauvres, et tout cela parce qu'il m'aura plu, un soir, de me battre avec Royat, (fig. $\mathbf{n}^{\circ}{ }^{13}$ ) qui est à droite, avec Châtel-Guyon qui est à gauche, avec Le Mont-Dore, La Bourboule.... J'en ai maintenant pour trois ans de plaisirs avec ma ville. ${ }^{5}$. 


\section{NOTES}

1. PENEZ, Jérôme. Histoire du thermalisme en France au XIXe siècle. Thèse de sociologie. Paris : Paris VII, 2000.

2. ROMAINS, Jules. Les hommes de bonne volonté. Tome 5 : «Les Superbes ». Paris : Laffont, 1988. P. 757-760.

3. BOUNEAU, Christophe. « La promotion du thermalisme par la Compagnie du Midi de 1852 à 1937 ». Villes d'eaux. Histoire du thermalisme. Congrès national des sociétés savantes, Clermont-Ferrand, Paris, 1994, CTHS. P. 359.

4. PENEZ, Jérôme. Dans la fièvre thermale. La Société des eaux minérales de Châtel-Guyon, 1878-1914. Réussite et expansion d'une entreprise thermale. Institut du Massif Central. Clermont-Ferrand : C.H.E.C., 1994. P. 63-65.

5. MAUPASSANT, Guy de. Mont-Oriol. P. 72-73.

\section{RÉSUMÉS}

Le XIXe siècle est le siècle de la « fièvre thermale » en France. Pour permettre cet extraordinaire développement, l'argent est nécessaire. La station thermale apparait comme un eldorado où la fortune est à portée de la main. Cette fortune n'est pourtant pas si facile à obtenir, la gestion thermale est ingrate, et les centaines de villes d'eaux françaises ne peuvent pas toutes obtenir un succès comparable à celui de Vichy ou d'Aix-les-Bains. Le thermalisme français est diversité, diversité dans les réussites, diversité dans les types de gestion (de la gestion étatique à la gestion privée), diversité dans sa fréquentation, diversité dans ses périodes de développement, diversité dans les moyens de son financement. Etudier l'importance ou au contraire la rareté des réseaux d'investissement dans le thermalisme permet de lever une partie du voile de l'histoire encore bien mystérieuse des villes d'eaux françaises. 


\section{INDEX}

Mots-clés : Thermalisme, Vichy, Aix-les-Bains, station thermale de La Celle-les-Eaux, Pouguesles-Eaux, Luxeuil, Plombières, Néris, Bourbon, l'Archambault, Bourbonne-les-Bains, Provins, Bagnères-de-Bigorre, Cauterets, Luz, Barèges, Capvern, Labassère, Eaux-Bonnes, Eaux-Chaudes, Cambo, Plombières, Médagues, Félix Robillot, Joseph Parisot, Charles Demande, Victor de Pruines, Auguste Lebobe, Georges Callou, docteur Arnal, Jean-Frédéric Possoz, Arthur Callou, Luchon, Royat, Bagnères-de-Bigorre, Saint-Sauveur, Evian, Thonon, Mont-Dore, La Chaldette, La Bourboule, Brides, Hybord, Victor-Emmanuel, le Mont-Dore, Etienne Lizet, Chabaud, Larrieu, thermes de Cauterets, Benjamin Dulau, Louis Bouloumié, Vittel, Vidart, Divonne, Auguste Badoit, Saint-Galmier, Koechlin Schwartz, Jules Evrard, Henri de Tricornot, Bagnoles-de-l'Orne, Richard, Georges Hartog, Enghien, Albert de Montry de Villemessant, Bressous, Lapostolet, Certeux, Alet, Allevard, Andabre, Châteldon, Contrexéville, Desaignes, Euzet, Fumades, Salins-du-Jura, SaintGervais, Spa, Vals, hôtels, casino, Trouville, Brides-les-Bains, Antoine Callou, Isaac Péreire, Amélie-les-Bains, Jacques Vernes, Compagnie du chemin de fer du midi, Contrexéville, Achille Fould, Fauconnet, Alfred André, Baraduc, François Brocard, docteur Angelby, docteur Pessez, Emile Dussargues de Colombier, Brocard, François Brocard comte de Pontgibaud, Chalus et frères

\section{AUTEUR \\ JÉRÔME PENEZ}

Professeur agrégé d'histoire, docteur en histoire contemporaine.j.penez@wanadoo.fr 